\section{Delivery of a nematocyst toxin}

SIR - Cnidaria (hydras, jellyfish, sea anemones and corals) possess a wide array of neurotoxic, cytolytic and enzymatic ${ }^{1}$ substances. Despite the fact that these toxic substances were isolated mainly from homogenates of entire animals or tentacles, their storage and delivery sites are attributes to nematocysts, the stinging subcellular organelles. Nematocysts consist of a capsule containing a highly folded eversible tubule ${ }^{2}$. The discharge of the nematocysts is driven by

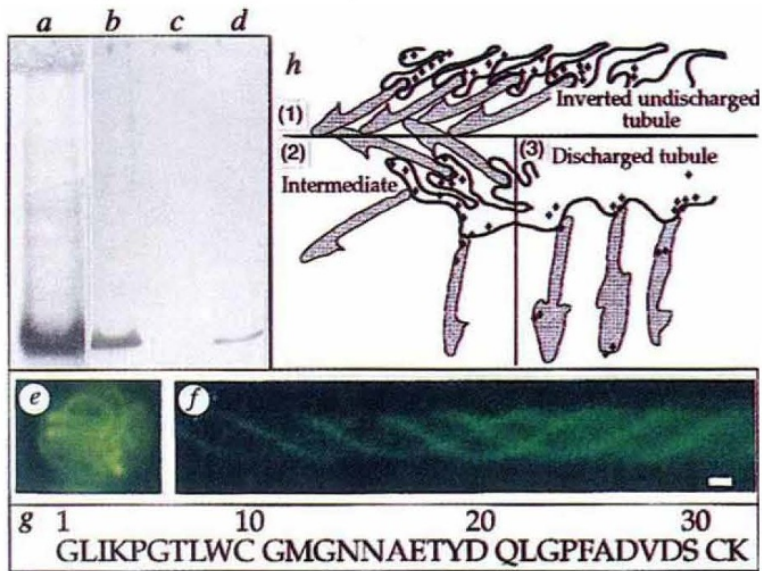

Chemistry, location and delivery of a nematocyst toxin derived from $R$. nomadica. a, SDS-PAGE electrophoresis of $2 \mu \mathrm{g}$ toxin. $\boldsymbol{b}-\boldsymbol{d}$, immunoblots. Separation of $10 \mu \mathrm{g}$ proteins extracted from the fishing tentacies $(b)$, bell tissues $(c)$ and $0.1 \mu \mathrm{g}$ of purified toxin $(d)$. Tentacles of the freshly collected jellyfish were cut at sea, stored over dried ice, then deep-frozen $\left(-80^{\circ} \mathrm{C}\right)$. Tentacles were thawed at $25^{\circ} \mathrm{C}$ followed by centrifugation $(15,000 \mathrm{~g}, 30 \mathrm{~min})$. The supernatant-containing material from discharged nematocysts was desalted by dialysis and separated by DEAE-cellulose anion-exchange column followed by Mono-S-HPLC cation-exchange column, resulting in a substance toxic to fishes by injection $\left(L_{50}=0.6 \mu \mathrm{g}\right.$ per $100 \mathrm{mg}$ body weight). Purity of the toxic component was assessed by electrophoresis, amino-acid analysis and $\mathrm{N}$-terminal aminoacid sequence determination. Proteins extracted from jellyfish tentacles were separated on a BioRad minigel apparatus, transferred to nitrocellulose membranes overlaid with the toxin-specific antibodies and detected by chemiluminescence. The western blotting kit (rabbit) was purchased from Boehringer-Mannheim. e, f, Immunofluorescent light microscopy of a resting $(e)$ and discharged $(f)$ nematocyst. Note that the stain revealing the toxin occurs in the tubule $(e)$ and is limited to its helically arranged dots $(f)$ when discharged. Bars: e, $2 \mu \mathrm{m} ; f, 1 \mu \mathrm{m}$. For light microscopy, tentacles were sliced by a Jung CM 3000 (Leica) cryostat to sections of $8 \mu \mathrm{m}$, attached to glass slides with polylysine, incubated with the affinity-purified antitoxin antibody, then with goat anti-rabbit fluorescein isothiocyanate-labelled antibody (Jackson Inc.). g, $\mathrm{N}$-terminal amino-acid sequence of the new toxin. This seg ment has about $60 \%$ amino-acid homology with toxic phospholipases $A_{2}$ derived from the venom of the Heloderma lizard and a honey bee ${ }^{5}$. $\boldsymbol{h}$, Schematic presentation of toxin compartmentation and delivery in the nematocyst system. (1), Resting tubule, whose lumen is filled with baros. The toxin (diamonds) is located in the folds and invaginations of the tubule's membrane (black line). (2), During discharge, toxin is translocated into the tubule while the barbs emerge and extend. (3), Toxin delivered from the everted-extended tubule through the hollow barbs. toxin on the discharged tubule revealed a helical shape $(f)$ which parallels the helical arrangement of the barbs. The latter observation has raised the possibility that the hollow barbs ${ }^{8}$ may function as a device for toxin delivery. This essential aspect was further studied by high-resolution immunogold technique and electron microscopy ${ }^{7}$. It has been shown that the toxin is externally allocated on the surface of the inverted-undischarged tubule. In the everted-discharged tubule the toxin occurs on its internal surface and accumulates in the vicinity of the bases of the hollow barbs and their lumina (data not shown).

The above data provide a visualization of the route of toxin translocation and release schematically presented in $h$. In the resting nematocyst, toxin is located in the crypts (invaginations) of the outer surface of the twisted, folded and inverted tubule, the lumen of which is occupied by the internalized barbs. During discharge and tubule eversion toxin is translocated from the outer to the inner surface of the everted and extended tubule while aggregating near the bases of and within the hollow barbs during release.

We assume that the hydrostatic pressure raised in the dischargeable capsule $^{9}$, causing the tubule eversion, also supplies the appropriate propulsion of toxin delivery through the barb system. This notion is supported by a study showing that the fibre-like structure of the capsule's inner wall, which provides the tensile strength necessary to withstand the capsule's high osmotic pressure, continues along the tubule's wall ${ }^{3}$. Thus, the nematocyst delivery system may provide an example of subcellular protein translocation based on simple mechanical forces.

\section{A. Lotan ${ }^{\star \dagger}$}

\section{Fishman*}

Y. Loya ${ }^{\dagger}$

\section{E. Zlotkin}

"Department of Cell and Animal Biology, Life Science Institute,

Hebrew University,

Jerusalem 91904, Israel

${ }^{+}$Department of Zoology,

Faculty of Life Sciences,

Tel Aviv University,

Tel Aviv 69978, Israel

and is not likely to be widely distributed defence chemical.

Immunocytochemical staining of cryostat-sliced nematocysts revealed a highly specific allocation of the toxin limited to their tubular segments $(e, f)$. In the resting nematocyst $(e)$ the capsular lumen and wall were devoid of immunofluorescent staining. The allocation of the
1. Walker, M. J. A. in Marine Toxins and Venoms (ed. Tu, A. T.) 279-325 (Dekker, New York, 1988).

2. Mariscal, R. N. in Coelenterate Biology (eds Muscatine, L. \& Lenhof, H. M.) 129-178 (Academic, New York, 1974)

3. Holstein, T. W. et al. Science 265, 402 (1994).

4. Holstein, T. W. \& Tardent, P. Science 223, 830 (1984)

5. Gomez, F. et al. Eur. J. Biochem. 186, $23-33$ (1989)

6. Vaitukaitis, J. L. Meth. Enzym. 73, 46-52 (1981).

7. Castel, M., Belenky, M., Cohen, S., Otterson, 0. \& Mathisen, J. S. Eur. J. Neurosci. 5, 368 (1993).

8. Hessinger, D. A. \& Ford, M. T. in The Biology of Nematocysts (eds Hessinger D. A. \& Lenhoff, H. M.) 75-94 (Academic, Orlancio, 1988).

9. Tardent, P. in The Biology of Nematocysts (eds Hessinger, D. A. \& Lenhoff, H. M.) 309-332 (Academic, Orlando, 1988). 